\title{
Quantum vortices in optical lattices
}

\author{
P. Vignolo, ${ }^{1,2}$ R. Fazio, ${ }^{3,1}$ and M.P. Tosi ${ }^{1}$ \\ ${ }^{1}$ NEST-CNR-INFM and Scuola Normale Superiore, I-56126 Pisa, Italy \\ ${ }^{2}$ INFN, largo B. Pontecorvo 3, I-56127 Pisa, Italy \\ ${ }^{3}$ International School for Advanced Studies (SISSA), via Beirut 2-4, I-34014 Trieste, Italy
}

\begin{abstract}
A vortex in a superfluid gas inside an optical lattice can behave as a massive particle moving in a periodic potential and exhibiting quantum properties. In this Letter we discuss these properties and show that the excitation of vortex motions in a two-dimensional lattice can lead to striking measurable changes in its dynamic response. It would be possible by means of Bragg spectroscopy to carry out the first direct measurement of the effective vortex mass, the pinning to the underlying lattice, and the dissipative damping.
\end{abstract}

PACS numbers: 05.30.Jp; 74.78.w; 74.81.Fa

The understanding of the static and dynamical behavior of vortices has been crucial to describe numerous different situations in superfluids ranging from liquid Helium to high-temperature superconductors [1, 2]. These defects can be created by means of an applied magnetic field in superconductors and by putting the sample into rotation in superfluid Helium, or they can be thermally excited in low-dimensional systems where the unbinding of vortex-antivortex pairs is at the core of the BerezinskiiKosterlitz-Thouless transition. Low-dimensional superconductors and in particular Josephson Junction Arrays (JJAs) have been for many years a natural playground for studying classical and quantum properties of vortices [3]. A vortex in a JJA behaves as a massive particle moving in a periodic potential and subject to dissipation [4], and under appropriate conditions vortices can show quantum properties such as interference or tunneling. Among the most interesting experiments performed with vortices in JJAs we mention the observation of ballistic motion [5], the measurement of the Aharonov-Casher effect for a vortex going around a charge [6], and the Mott-Anderson insulator of vortices [7].

Optical lattices for atomic gases, which currently are under intense investigation [8, 9, 10], can behave as tunneling junction arrays. In this Letter we analyze vortex excitations in an optical lattice and show that a superfluid gas in an optical lattice offers a unique opportunity for a direct measurement of vortex properties (such as the mass, the coupling to its environment or the pinning potential) via a Bragg spectroscopy experiment. This is in contrast to the JJA case, where only indirect measurements based on transport properties are available. The Bragg spectroscopy technique [11, 12, 13] has been appealed to for a variety of experiments on ultra-cold atomic gases, and in optical lattices has been considered for a measurement of the excitation spectrum of a Bose gas in the Mott-insulator phase [14], and of its coexistence with a superfluid phase in a dishomogeneous cloud [15].

We consider a Bose gas in the superfluid phase inside a lattice [16, 17], in a regime where the hopping and the on-site repulsion between the bosons are competitive. Quantum fluctuations due to the interplay of the local repulsions and of the hopping have dramatic consequences for vortex dynamics. In this case a vortex behaves as a macroscopic quantum particle, moving in a periodic potential with a mass that we evaluate and show to be directly measurable by Bragg spectroscopy. At variance from other recent studies of vortices in frustrated optical lattices 18, 19, 20, 21, 22, 23], we discuss the dynamical properties of an individual vortex. In order to achieve this regime one can either apply a very low frustration by means of a rotation of the lattice [24] generating only a few and very weakly interacting vortices, or create a vortex excitation by means of phase inprinting [25, 26]. Of particular relevance is the very recent observation of vortex pinning in co-rotating optical lattices by Tung et al. [24], which indicates that what we propose here is within reach of experimental verification.

The model - We consider a Bose gas at zero temperature inside a square lattice with lattice constant $a$ and $N_{s}$ lattice sites. We assume that the system can be described by a single-band Bose-Hubbard Hamiltonian [27]

$$
H=-\frac{J}{2} \sum_{\langle i j\rangle} \hat{b}_{i}^{\dagger} \hat{b}_{j}+\text { H.c. }+U \sum_{i} \hat{n}_{i}\left(\hat{n}_{i}-1\right)-\mu \sum_{i} \hat{n}_{i},
$$

where $\hat{b}_{i}^{\dagger}$ and $\hat{b}_{i}$ are the creation and annihilation operators for a boson on the $i$-th site and $\hat{n}_{i}=\hat{b}_{i}^{\dagger} \hat{b}_{i}$ is the number operator. The coupling constant $U$ describes the local interaction between bosons, $\mu$ is the chemical potential, and $J$ the matrix element for hopping between nearestneighbors sites. The on-site interaction energy and the hopping energy are given by $U=g \int d \mathbf{r}\left|w_{0}\left(\mathbf{r}-\mathbf{R}_{i}\right)\right|^{4}$, $J=-\left(\hbar^{2} / 2 m\right)^{-1} \int d \mathbf{r} w_{0}^{*}\left(\mathbf{r}-\mathbf{R}_{i}\right) \nabla^{2} w_{0}\left(\mathbf{r}-\mathbf{R}_{j}\right)$, in terms of the Wannier function $w_{0}(\mathbf{r})\left(\mathbf{R}_{i}\right.$ is the coordinate of the $i$-th site). Here $g=4 \pi \hbar^{2} a_{s c} /\left(\sqrt{2 \pi} m l_{\perp}\right)$ is the repulsive interaction strength in the case where the transverse size $l_{\perp}$ of the lattice is larger than the scattering length $a_{s c}$.

If the average number of bosons per site is much larger than one, the field operators can be approximated as $\hat{b}_{i} \simeq$ 
$\sqrt{\bar{n}} \exp \left(i \hat{\phi}_{i}\right)$, with $\hat{\phi}_{i}$ being the phase operator on the $i$ th site. The Bose-Hubbard model can be recast into the quantum phase Hamiltonian

$$
\hat{H}=-J \bar{n} \sum_{\langle i, j\rangle} \cos \left(\hat{\phi}_{i}-\hat{\phi}_{j}\right)+U \sum_{i} \delta \hat{n}_{i}^{2}-\tilde{\mu} \sum_{i} \delta \hat{n}_{i}
$$

where $\tilde{\mu}=2 U-\mu-1$. The number operator has been expressed in terms of the fluctuations around its average value $\bar{n}, \hat{n}_{i}=\bar{n}+\delta \hat{n}_{i}$. The number fluctuation operator and the phase are canonically conjugate variables, $\left[\delta \hat{n}_{i}, e^{ \pm i \phi_{j}}\right]=\delta_{i j} e^{ \pm i \phi_{j}}$. The regime that we consider throughout this work is $J \bar{n} \gg U$ : the system is deep in the superfluid region, but quantum fluctuations are present and play a crucial role in the vortex dynamics.

Vortex properties - The presence of a static vortex inside the lattice can be described to a good approximation by a phase distribution of the boson field given by

$$
\phi_{i}=\arctan \left(\frac{y_{i}-y}{x_{i}-x}\right),
$$

where $x, y$ are the coordinates of the center of the vortex. Deep in the superfluid regime and at temperatures much lower than $J \bar{n} / K_{B}$, phase rigidity ensures that again to a good approximation, a moving vortex can still be described by Eq. (3) but with a time-dependent position of the vortex center. The existence of a vortex mass can be understood qualitatively by noting that if a vortex moves of a distance of the order of $a$ in a time $\delta t=a / v$, $v$ being its velocity, the phase difference $\delta \phi_{i j}$ at each bond changes in time as $\delta \phi_{i j}=\phi_{i j}(t+a / v)-\phi_{i j}(t)$. Due to the commutation relation between the number and phase operators, a time-dependent phase leads to a contribution to the energy which is quadratic in the vortex velocity (see the second term of the r.h.s of Eq.(2)). The problem of calculating the vortex mass can then be reduced to find the phase differences across junctions at times $t$ and $t+a / v$.

An effective action for a vortex in a lattice can then be obtained by inserting Eq. (3) in the Bose-Hubbard model in Eq. (2) and then expressing the resulting action in terms of the vortex coordinates $\mathbf{r}(t)=\{x(t), y(t)\}[4]$. The on-site repulsion term provides a kinetic energy term $T=\left(M_{v} / 2\right) \dot{\mathbf{r}}^{2}$, where the vortex mass in a lattice of size $L$ is

$$
M_{v}=\frac{\sqrt{2 \pi} l_{\perp} w^{2}}{4 a_{s c} a^{2}} m \ln (L / a) .
$$

The vortex mass thus scales linearly with the boson mass, increases with the width $w$ of the Wannier function, and decreases with the scattering length.

Let us consider for an illustration the ${ }^{87} \mathrm{Rb}$ lattice realized by Greiner and coworkers [17], in the case $V_{0}=4 E_{r}$ where $V_{0}$ and $E_{r}$ are the well depth of the optical lattice and the recoil energy respectively. For such a system in $2 \mathrm{D}$ we have $a=426 \mathrm{~nm}, w \simeq 96 \mathrm{~nm}, l_{\perp}=5 \mu \mathrm{m}$, $a_{s c}=5.5 \mathrm{~nm}$ and $L=75 \mu \mathrm{m}$, and the vortex mass is

$$
M_{v} \simeq 29 m \ln (L / a) \simeq 150 m \simeq 2.2 \times 10^{-20} \mathrm{gr} .
$$

The behavior of the vortex mass as a function of the potential well depth (which affects $w$ ) and of the size of the lattice is depicted in Fig. 1,

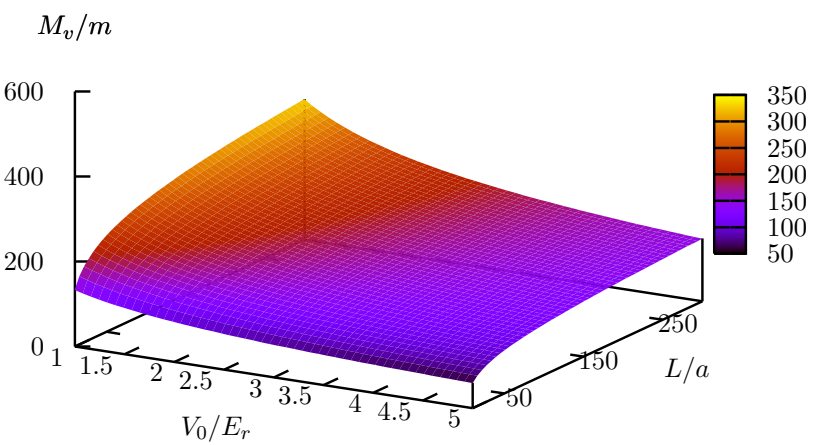

FIG. 1: The vortex mass $M_{v}$ (in units of the boson mass $m$ ) as a function of $V_{0} / E_{r}$ and $L / a$, for the case $a=426 \mathrm{~nm}$, $l_{\perp}=5 \mu \mathrm{m}$, and $a_{s c}=5.5 \mathrm{~nm}$.

The effective potential seen by the vortex has been numerically evaluated in the context of JJAs and, in the case of a vortex moving in the $x$ direction inside a large two-dimensional array the effective potential is periodic [28],

$$
U_{v}(x)=0.1 J \bar{n}[\cos (2 \pi x / a)-1] .
$$

In the presence of a vortex the whole array thus behaves as a macroscopic particle of mass $M_{v}$ moving in a periodic potential. For such a macroscopic object one has to also take into account the interaction with the environment, and the main source of damping is due to the interaction with the long-wavelength phase modes that are excited during vortex motion. This damping has been analyzed in detail in the context of JJAs (see for example [29, 30]). In this Letter for simplicity we shall just comment on how our results are modified in accounting for dissipation.

Dynamic structure factor - We turn to a calculation of the dynamical response of the Bose gas inside a lattice, both in the absence and in the presence of a vortex. The central quantity of interest is the dynamic structure factor, which in a tight-binding scheme takes the form

$$
S(\mathbf{q}, \omega)=\int d t e^{i \omega t} \sum_{i, j} e^{-i \mathbf{q} \cdot\left(\mathbf{R}_{i}-\mathbf{R}_{j}\right)}\left\langle\delta \hat{n}_{i}(t) \delta \hat{n}_{j}(0)\right\rangle .
$$

This spectrum can be measured in experiments of Bragg spectroscopy [11, 12]: two probe laser beams, with frequencies $\omega_{1}$ and $\omega_{2}=\omega_{1}+\omega$ and wave-vectors $\mathbf{k}_{1}$ and $\mathbf{k}_{2}=\mathbf{k}_{1}+\mathbf{q}$, scatter on the boson gas and the 
spectrum measures the probability of momentum transfer $\hbar \mathbf{q}$ at energy $\hbar \omega$ [13]. The $f$-sum rule gives the first spectral moment $M_{1}(\mathbf{q})$ as $M_{1} \equiv \int S(\mathbf{q}, \omega) \omega d \omega=$ $\frac{1}{2 \hbar}\langle 0|\left[\delta \hat{n}_{\mathbf{q}},\left[\hat{H}, \delta \hat{n}_{\mathbf{q}}^{\dagger}\right]|0\rangle, \delta \hat{n}_{\mathbf{q}}\right.$ being the Fourier transform of $\delta \hat{n}_{i}$.

We first consider the case in which no vortex is present. Inside the superfluid regime $(J \bar{n} \gg U)$, it is enough to consider long-wavelength phase fluctuations, as described by expansion of the cosine in the phase Hamiltonian up to second order. In this limit the Hamiltonian is easily diagonalized in Fourier space by means of the transformations $\hat{\phi}_{\mathbf{k}}=\left[U N_{s} /\left(\hbar \Omega_{\mathbf{k}}\right)\right]^{1 / 2}\left(\hat{a}_{\mathbf{k}}+\hat{a}_{-\mathbf{k}}^{\dagger}\right) / \sqrt{2}$ and $\hat{n}_{\mathbf{k}}=\left(N_{s} \hbar \Omega_{\mathbf{k}} / U\right)^{1 / 2}\left(\hat{a}_{\mathbf{k}}-\hat{a}_{-\mathbf{k}}^{\dagger}\right) / i \sqrt{2}$, with the result

$$
\hat{H}=\sum_{\mathbf{k} \in \mathrm{BZ}} \hbar \Omega_{\mathbf{k}}\left(\hat{a}_{\mathbf{k}}^{\dagger} \hat{a}_{\mathbf{k}}+\frac{1}{2}\right)
$$

where $\Omega_{\mathbf{k}}^{2}=\left(2 J \bar{n} U / \hbar^{2}\right)\left[2-\cos \left(k_{x} a\right)-\cos \left(k_{y} a\right)\right]$. Here the quasi-momentum $\mathbf{k}=\left(k_{x}, k_{y}\right)$ is inside the first Brillouin zone. By taking into account the time dependence of the particle number fluctuation operator dictated by Eq. (8), it is straightforward to obtain the dynamic structure factor as

$$
S(\mathbf{q}, \omega)=\frac{\hbar \Omega_{\mathbf{q}}}{2 U} \delta\left(\omega-\Omega_{\mathbf{q}}\right) .
$$

The physical interpretation of Eq. (9) is clear: small- $q$ absorption occurs at a frequency corresponding to the dispersion relation of the Goldstone sound mode in the lattice. In this case $M_{1}(\mathbf{q})=(J \bar{n} / \hbar)\left[2-\cos \left(q_{x} a\right)-\right.$ $\left.\cos \left(q_{y} a\right)\right]$.

The presence of a vortex can induce, besides changes in the sound wave spectrum, specific contributions associated with excitations of vortex motions. Several different situations can be envisaged for the latter, but here we discuss the interesting case in which the hopping parameter $J$ is sufficiently large that the vortex is pinned to a minimum of the periodic potential given by Eq. (6). In this case the vortex dynamics is associated with small oscillations around its equilibrium position and can be described by the harmonic oscillator Hamiltonian

$$
H_{v}=\frac{1}{2} M_{v} \dot{\mathbf{r}}^{2}+\frac{1}{2} M_{v} \Omega_{v}^{2} \mathbf{r}^{2},
$$

where we have defined $\Omega_{v}=\left(0.1 J \bar{n} / M_{v}\right)^{1 / 2} 2 \pi / a$. By noting that

$$
\left\langle\delta \hat{n}_{i}\left(t_{1}\right) \delta \hat{n}_{j}\left(t_{2}\right)\right\rangle=\frac{U^{2}}{\hbar^{2}}\left\langle\delta \hat{\dot{\phi}}_{i}\left(t_{1}\right) \delta \hat{\dot{\phi}}_{j}\left(t_{2}\right)\right\rangle,
$$

using the expression given in Eq. (3) for the phase distribution and performing an average over the vortex degrees of freedom with the help of Eq. (10), it is possible to write the contribution of the vortex to the dynamic structure factor as

$$
S_{v}(\mathbf{q}, \omega)=\frac{\hbar^{2} \Omega_{v}}{U^{2}} \frac{4 \pi^{2} \hbar}{M_{v} a^{4} q^{2}} \delta\left(\omega-\Omega_{v}\right)
$$

Instead of a q-dependent resonance as in Eq.(9), the Bragg scattering acquires a resonance at a well defined frequency $\Omega_{v}$ indicating that the whole lattice responds collectively having the properties of a single macroscopic particle, the vortex. This is the main result of this paper. Under the conditions specified above, the presence of a vortex induces a resonance at a frequency that allows access to the vortex mass. Let us remark that this resonant behavior is related to the presence of the lattice and to the existence of quantum fluctuations originating from the local repulsion. The Bragg spectrum of a vortex in a Bose-Einstein condensate is otherwise determinated by a dispersion relation [13]. The peculiar dependence of the spectral strength in Eq. (12) on the transferred momentum $q$ is due to the coupling between the exciting radiation and the lattice: at low momentum all phases in the lattice are excited and the dynamic response is enhanced. We finally should comment on the fact that Eq. (12) does not fulfill the $f$-sum rule: this should come as no surprise, as this expression is valid only at low energy.

The coupling to long-wavelength phase fluctuations provides the main dissipation mechanism for the vortex motions [29, 30, 31]. To a first approximation this results in Ohmic damping on the vortex. In the presence of dissipation the delta function in the dynamical response is smeared and acquires a finite width proportional to the dissipation strength.

In summary, in this Letter we have discussed some main aspects of quantum dynamics of a vortex in an atomic superfluid gas inside an optical lattice. We have specifically considered the situation in which the vortex is pinned by the lattice potential and only executes small oscillations around its energy minimum. In this case Bragg spectroscopy should allow a measurement of the vortex mass. One can envisage other situations in which to study vortex dynamics. Experiments on quantum tunneling/coherence of vortices seem to be within experimental reach.

We acknowledge fruitful discussion with C. Bruder and M. Polini. This work has been supported by MIUR-PRIN and by EC through grants EUROSQIP and RTNNANO.

[1] G. E. Volovik, The Universe in a Helium droplet (Clarendon Press, Oxford, 2003).

[2] M. Tinkham, Introduction to Superconductivity (McGraw-Hill, New York, 1996).

[3] R. Fazio and H.S.J. van der Zant, Phys. Rep. 355, 235 (2001).

[4] U. Eckern and A. Schmid, Phys. Rev. B 10, 6441 (1989).

[5] H.S.J. van der Zant, F.C. Fritschy, T.P. Orlando, and J.E. Mooij, Phys. Rev. Lett. 66, 2531 (1991).

[6] W.J. Elion, M. Matters, U. Geigenmüller, and J.E. Mooij, Phys. Rev. Lett. 71, 2311 (1993). 
[7] A. van Oudenaarden and J.E. Mooij, Phys. Rev. Lett. 76, 4947 (1996); A. van Oudenaarden, S.J.K. Várdy, and J.E. Mooij, Phys. Rev. Lett. 77, 4257 (1996).

[8] A. Minguzzi, S. Succi, F. Toschi, M.P. Tosi, and P. Vignolo, Phys. Rep. 395, 223 (2004).

[9] O. Morsch and M. Oberthaler, Rev. Mod. Phys. 78, 179 (2006).

[10] M. Lewenstein, A. Sanpera, V. Ahufinger, B. Damski, A. Sen(De), and U. Sen, cond-mat/0606771

[11] J. Stenger, S. Inouye, A.P. Chikkatur, D.M. StamperKurn, D.E. Pritchard, and W. Ketterle, Phys. Rev. Lett. 82, 4569 (1999).

[12] D.M. Stamper-Kurn, A.P. Chikkatur, A. Gorlitz, S. Inouye, S. Gupta, D.E. Pritchard, and W. Ketterle, Phys. Rev. Lett. 83, 2876 (1999).

[13] P.B. Blackie, R.J. Ballagh and C.W. Gardiner, Phys. Rev. A 65, 033602 (2002).

[14] A.M. Rey, P.B. Blackie, G. Pupillo, C.G. Williams, and C.W. Clark, Phys. Rev. A 72, 023407 (2005).

[15] G.G. Batrouni, F.F. Assaad, R.T. Scalettar, and P.J.H. Denteneer, Phys. Rev. A 72, 031601(R) (2005).

[16] D. Jaksch, C. Bruder, J.I. Cirac, C.W. Gardiner, and P. Zoller, Phys. Rev. Lett. 81, 3108 (1998).

[17] M. Greiner, O. Mandel, T. Esslinger, T.W. Hänsch, and I. Bloch, Nature 415, 39 (2002).

[18] D. Jaksch and P. Zoller, New J. Phys. 5, 56 (2003).
[19] A.S. Sørensen, E. Demler, and M.D. Lukin, Phys. Rev. Lett. 94, 086803 (2005).

[20] M. Polini, R. Fazio, A.H. MacDonald, and M.P. Tosi, Phys. Rev. Lett. 95, 010401 (2005).

[21] E.J. Mueller, Phys. Rev. A 70, 041603(R) (2004).

[22] R. N. Palmer and D. Jaksch, Phys. Rev. Lett. 96, 180407 (2006).

[23] K. Osterloh, M. Baig, L. Santos, P. Zoller, and M. Lewenstein, Phys. Rev. Lett. 95, 010403 (2005).

[24] S. Tung, V. Schweikhard, and E.A. Cornell Phys. Rev. Lett. 97, 240402 (2006).

[25] M.R. Matthews, B.P. Anderson, P.C. Haljan, D.S. Hall, C.E. Wieman, and E.A. Cornell, Phys. Rev. Lett. 83, 2498 (1999).

[26] L. Dobrek, M. Gajda, M. Lewenstein, K. Sengstock, G. Birkl, and W. Ertmer, Phys. Rev. A 60, 3381(R) (1999).

[27] M.P.A. Fisher, P.B. Weichman, G. Grinstein, and D.S. Fisher, Phys. Rev. B 40, 546 (1989).

[28] C.J. Lobb, D.W. Abraham, and M. Tinkham, Phys. Rev. B 27, 150 (1983).

[29] U. Geigenmüller, C.J. Lobb, and C.B. Whan, Phys. Rev. B 47, 348 (1993).

[30] R. Fazio, A. van Otterlo, and G. Schön, Europhys. Lett. 25, 453 (1994).

[31] U. Eckern and E.B. Sonin, Phys. Rev. B 47, 505 (1993). 